\title{
The effect of painful laser stimuli on EEG gamma-band activity in migraine patients and healthy controls
}

lege Bassez ${ }^{a}$, Katia Ricci ${ }^{b}$, Eleonora Vecchio ${ }^{b}$, Marianna Delussi $^{b}$, Eleonora Gentile ${ }^{b}$, Daniele Marinazzo ${ }^{a}$, Marina de Tommaso ${ }^{b}$

a Department of Data Analysis, Ghent University, Ghent, Belgium

Postal address: Henri Dunantlaan 2, 9000 Gent, België

Corresponding author: lege.Bassez@UGent.be

Daniele.Marinazzo@UGent.be

${ }^{\mathrm{b}}$ Applied Neurophysiology and Pain Unit, SMBNOS Department, Polyclinic General Hospital, Bari Aldo Moro University, Bari, Italy

Postal address: Via Amendola 207 A, 70123 Bari, Italy

katiari86@gmail.com

eleonora.vecchio@gmail.com

m.delussi@gmail.com

eleonora.gentile@uniba.it

marina.detommaso@uniba.it 


\begin{abstract}

\section{Objective}

Gamma-band oscillations (GBOs) induced by nociceptive stimuli were compared between migraine and control in order to further characterize pain processing in the brain of interictal migraineurs. GBOs were related to subjective pain intensity, years of migraine history and migraine attack frequency and the sources of GBOs were investigated.
\end{abstract}

\title{
Methods
}

Twenty-three migraine patients without aura and 23 controls received a series of laser stimulations on their right forehead and right hand while recording electroencephalographic data (61 electrodes). After each series they indicated the perceived pain. A multitaper timefrequency method was used on artifact-cleaned scalp data and frequency domain beamforming was used to localize the GBOs.

\section{Results}

In both groups we observed increases in GBOs around central electrodes, which were not significantly different between groups. The central GBOs were positively associated with the subjective pain ratings in the control group, in accordance with previous studies, but not in the migraine group. Increases in gamma power were observed in the midcingulate cortex.

\section{Conclusions}

No evidence was found that GBOs differ between interictal migraine and controls nor that central GBOs represent a neurophysiological correlate of subjective pain in migraine.

\section{Significance}

We shed light on observations of GBOs during pain processing in interictal migraine.

\section{Highlights}

- Central GBOs seem to be related to pain perception in controls but not in migraine.

- No differences found in nociceptive-related GBOs between interictal migraine patients and controls.

- We suggest the midcingulate cortex to be a source of nociceptive-related GBOs

Keywords: EEG, thermonociceptive stimulation, pain, gamma-band oscillations, highfrequency neural activity, migraine 


\section{Introduction}

Previous studies using different brain functional analysis methods showed atypical pain processing in interictal migraine compared to control groups. This has been found with functional magnetic resonance imaging ( $\mathrm{fMRI}$ ) where impaired habituation to repeated painful stimuli has been found in the bilateral anterior insula, the midcingulate cortex and the thalamus (Stankewitz et al., 2013) as well as atypical activation of brain regions during painful stimulation (Schwedt et al., 2015). Using resting-state (RS) fMRI, several studies have shown that some intrinsic functional connections between regions in RS networks differ in migraine patients compared to healthy controls (Colombo et al., 2015). Colombo et al. (2015) summarized these findings, noting that in areas related to nociception, RS functional connectivity is mostly increased in migraine patients compared to healthy controls. In contrast, between regions related to pain modulation, RS functional connectivity seems to be mostly decreased. Several studies using electroencephalography (EEG) have also shown reduced habituation to repeated painful laser stimuli in migraine (de Tommaso et al., 2014; Valeriani et al., 2003). As interictal atypical pain processing in migraine may possibly indicate the readiness of the migrainous brain to generate attacks (Goadsby et al., 2017) it is important to further characterize the extent and features of this atypical interictal pain processing.

Most EEG studies examining pain processing in migraine using laser stimuli have looked at the laser-evoked potential (LEP) components (i.e., N1, N2, P2). However, several studies show that LEPs are not a signature of pain perception but rather reflect stimulus saliency (lannetti et al., 2008; Ronga et al., 2013). Although LEPs are very useful to study the nociceptive system (Treede et al., 2003), we could possibly improve the study of the nociceptive system in migraine by looking at other EEG features induced by laser stimuli. For example, in the pain literature, several studies have found an increase in high-frequency oscillations (gamma-band) between 150 and 350 milliseconds after painful laser stimulations (Ploner et al., 2017). In EEG research, these gamma-band oscillations (GBOs) are mostly found between 70 and $90 \mathrm{~Hz}$ at central electrodes (Schulz et al., 2011a, 2011b, 2012). In addition to being related to pain perception (Gross et al., 2007; Schulz et al., 2011b; Zhang et al., 2012), it has been shown that GBOs assumed to be generated by the primary somatosensory region could reflect pain perception, rather than stimulus saliency (Zhang et al., 2012). An intracerebral EEG study showed that GBOs recorded from the insula are preferential for nociception as insular GBOs were more pronounced after painful laser stimulations than after equally arousing visual, auditory and vibrotactile stimuli (Liberati et al., 2018a). However, unlike GBOs recorded over the primary somatosensory region (Zhang et al., 2012), the insular GBOs can be dissociated from pain perception as insular GBOs habituated to repetitive stimulations while intensity ratings did not (Liberati et al., 2018b).

In the current study, we investigated GBOs related to nociception in episodic migraine patients without aura. The aims of the study were 1) to compare the increase in gamma-band power related to somatic and trigeminal laser stimulation between migraine without aura patients 
and controls, 2) to correlate the increase in GBOs at central electrodes with subjective pain ratings, 3 ) to correlate the increase in $\mathrm{GBO}$ s at central electrodes with years of migraine history and migraine attack frequency and 4) to localize the GBOs increases using a beamforming technique.

\section{Methods}

\subsection{Participants}

Twenty-three episodic migraine patients without aura (16 females, $M$ age $=35.13$ years, $S D=$ 12.57 years) were included in this study. Patients were diagnosed according to ICHD-3 criteria (IHS, 2013). Diagnoses were confirmed considering more recent criteria (IHS, 2018). The migraine patients in this study had a history of migraine attacks between 2 and 30 years $(M=$ 13.22 years, $S D=8.05)$. The mean headache frequency was 6.65 days with headache in a month $(S D=4.38)$. The pain intensity of migraine attacks that patients experienced varied from 6 to 10 with a mean of $9(S D=1.30)$ on a scale of 10 , indicating that most patients experienced severe painful migraine attacks. Patients were tested between headache attacks, at least 72 hours after the last attack, and more than 48 hours before the next one, thereby excluding patients in the phases preceding a migraine attack. The timing of their migraine attacks was ascertained by direct or telephone contact. Twenty-three healthy volunteers (16 females, $M$ age $=33.65$ years, $S D=13.88$ years) were selected based on the absence of personal and first-degree familiar history of migraine. The protocol for this neurophysiological study was approved by the Ethical Committee of Bari Policlinic General Hospital. All participants provided their written informed consent.

\subsection{EEG and Procedure}

The participants laid on a couch with their eyes open in a warm semi-darkened room. A 61 channels montage was used. The recording electrodes were placed on the scalp referred to the nasion, according to the extended international 10-20 System (i.e., 10-10 system). The recording system was a MICROMED EEG apparatus (Micromed Brain Quick, Mogliano Veneto, Italy). Two additional electrodes were positioned below the eyes for electrooculogram recording. The impedance was kept below $4 \mathrm{~K} \Omega$. During the recording sessions, digital filters in the $0.1-70 \mathrm{~Hz}$ range and a $50 \mathrm{~Hz}$ notch filter were applied to allow signal inspection. Participants received a series of 15 painful laser stimulations on the dorsum of the right hand and a series of 15 painful laser stimulations on the right forehead (corresponding to the first branch of the trigeminal nerve). Three of the control participants did not receive a series of painful stimuli on the forehead. The inter stimulation interval was self-paced and varied around 10 seconds. The laser stimulations were cutaneous heat stimuli delivered by a $\mathrm{CO} 2$ laser (wavelength: $10.6 \mathrm{~mm}$; beam diameter: $2 \mathrm{~mm}$; ELEN, Florence, Italy). To avoid damage to the skin, fatigue or sensitization of nociceptors, the irradiated spot was shifted after each stimulus. Before each series, the intensity ( $\min 6 \mathrm{Watt}$, max $9 \mathrm{Watt}$ ) and duration ( $\min 15 \mathrm{ms,}$ $\max 45 \mathrm{~ms}$ ) of the laser stimulations were adjusted so that the participants genuinely experienced the laser stimulations as painful. When the participants rated the stimuli as 
painful, the power was increased with one unit in order to be certain the stimuli exceeded participants' pain threshold. After each series, participants were asked to indicate the perceived pain during that series on a visual analogue scale (VAS) ranging from 0 to 100 . On the VAS, the white color corresponding to 0 indicates no pain sensation while the intense red corresponding to 100 indicates the worst pain conceivable.

\subsection{EEG Preprocessing}

The data were preprocessed in MATLAB with an automatic pipeline using EEGLAB (v14.1.1; Delorme and Makeig, 2004) and plug-in functions. The data were first high-passed filtered with a lower-cut off of $1 \mathrm{~Hz}$ in order to remove slow drifts. Next, a notch filter at $50 \mathrm{~Hz}$ (L: 48, $\mathrm{H}: 52$ ) and $100 \mathrm{~Hz}$ (L:99, H: 101) was applied to remove power line noise artifacts. Other notch filters $(63-66 \mathrm{~Hz}, 73-77 \mathrm{~Hz}, 104-108 \mathrm{~Hz}$ ) were used to remove environmental artifacts. We applied an automatic approach referred to as Artifact Subspace Reconstruction (ASR, clean_rawdata plugin for EEGLAB) to correct continuous data and reject bad channels and data segments (Chang et al., 2019; Mullen et al., 2015). All removed channels were subsequently interpolated, and the data were re-referenced to the average. The data were then epoched at 3 and 2 seconds before and after the laser stimulation onset. These relatively long epochs were chosen to allow later removal of edge artifacts that arise due to the timefrequency transformation and to have a baseline with minimum expectation reactions. After epochs extraction, independent component analysis (ICA) was performed (runica EEGLAB function, using the pca option because of the rank deficiency as a result of interpolating and average re-referencing). Artifactual components were then automatically removed by using a machine learning algorithm called Multiple Artifact Rejection Algorithm (MARA; Winkler et al. 2011). Components with a probability of being artifactual higher than .9 were removed. MARA has been shown to perform well in rejecting muscle artifact components (Winkler et al., 2011) which was important in the current study.

\subsection{Descriptive Analysis}

To assess the quality of the data, we plotted the grand average LEP at central electrodes for both groups. Additionally, we made topographical plots at the N2 and P2 latencies to see if the pain responses look as would be expected from previous research. This analysis was performed using the FieldTrip toolbox (Oostenveld et al., 2011).

\subsection{Time-Frequency Analysis}

A multitaper analysis with 3 tapers was used to obtain a time-resolved frequency representation of the data. The center frequencies considered, ranged from 30 to $100 \mathrm{~Hz}$, in steps of $1 \mathrm{~Hz}$. The sliding time window (sliding in steps of $50 \mathrm{~ms}$ ) length (in $\mathrm{ms}$ ) and the width of frequency smoothing $(\mathrm{Hz})$ changed as a function of the center frequencies (5 cycles/frequency and 0.4 *frequency, respectively). The multitaper method was chosen as the use of multiple tapers with different temporal features increases the signal-to-noise ratio of the frequency representation (Cohen, 2014). As high-frequency activity has a rather low 
signal-to-noise ratio, this method is often recommended in these situations (Cohen, 2014). For every subject, the mean over trials was calculated. Then, the power data was normalized by using a decibel $(\mathrm{dB})$ transform ( $\mathrm{dB}$ power $=10 * \log 10$ [power/average baseline power]). As baseline we considered the time between 2 and 1 seconds before laser onset in order to avoid expectation reactions and edge artifacts. Decibel change in power from baseline between 70 and $90 \mathrm{~Hz}$ was averaged for every subject to represent gamma-band activity. We averaged 70$90 \mathrm{~Hz}$ because this frequency range was more or less found in several studies (Schulz et al., 2011a, 2011b, 2012). This analysis was performed using the FieldTrip toolbox (Oostenveld et al., 2011).

\subsection{Statistical Analysis}

We tested at every timeframe from 0 to 1 second and every electrode whether this gammaband activity was significantly different from baseline activity (i.e., HO: dB change from baseline $=0$ ). Nonparametric permutation (1000 permutations, random sign swapping) onesample t-tests were used with the maximum statistic approach to correct for multiple testing. To compare migraine patients with healthy controls, nonparametric permutation (1000 permutations, randomly assigning group label) two-sample t-tests were used with the maximum statistic approach to correct for multiple testing. As ongoing pre-stimulus gammaband activity may influence pain perception (Ploner et al., 2017), we compared the gamma spectrum in baseline between the migraine patients and controls. We used the baseline average power that was used to perform the decibel transform (see section 2.5.). Groups were compared on all 61 channels using permutation two-sample t-tests (1000 permutations) with the maximum statistic approach for multiple testing correction. The average GBOs (between 70-90 Hz and 100-300 ms for trigeminal stimulation, and between 150-350 ms for hand stimulation) averaged over $\mathrm{Cz}$ and FCz (Schulz et al., 2011b; Tiemann et al., 2010) were then correlated with the VAS ratings using nonparametric Spearman's rank correlations. For the migraine group, we additionally correlated the GBOs increases with the average number of attack days per month, computed in the three months preceding the recording session and with the years of migraine history. The maximum statistic approach was used for multiple testing correction. The Spearman correlation measure was used as it requires no distributional assumptions about the data and can capture any monotonic relation and is thus not restricted to just linear associations. Monte Carlo permutations (1000 permutations) were used to calculate $p$-values. All these analyses were performed using the FieldTrip toolbox (Oostenveld et al., 2011).

\subsection{Source Reconstruction}

Frequency domain beamforming was performed by using the Dynamic Imaging of Coherent Sources (DICS) method (Gross et al., 2001). The frequency of interest was $80 \mathrm{~Hz}$, with a frequency smoothing of $32 \mathrm{~Hz}$ (in accordance with the time-frequency settings). A multitaper frequency transformation was used. The cross-spectral density matrices were calculated for pre (-1800 to $-1600 \mathrm{~ms}$ ) and post (100 to $300 \mathrm{~ms}$ for forehead condition, $150-350 \mathrm{~ms}$ for hand 
condition) laser data (same length for pre and post laser time window), as well as for the appended pre and post data. First, a common spatial filter was calculated on the appended data which was then applied to the pre and post laser data separately. The forward model was calculated using a realistically shaped three-layer boundary element volume conduction model (BEM), on a 3-dimensional grid of dipole locations with equidistant spacing of $8 \mathrm{~mm}$. The Colin27 was used as MRI template. The automated anatomical labeling atlas which uses macroscopic parcels was used to label the sources (Tzourio-Mazoyer et al., 2002). The post source activity was contrasted against the pre source activity (pre-post/pre) and a thresholding mask was used to highlight those voxels with the $1 \%$ highest power increases.

\section{Results}

\subsection{Preprocessing}

Details on the performance of the preprocessing pipeline can be found in the supplementary material. Specifically, examples of ASR performance and artifactual muscle components can be found as well as a report per subject and per condition on the final number of trials and interpolated channels. GBOs are presented before and after ASR and ICA correction. In addition, the current preprocessing pipeline was compared with a pipeline where notch filters were replaced with a spectrum interpolation (Leske and Dalal, 2019) method. GBOs after the two preprocessing pipelines were not significantly different, suggesting robustness of the findings.

\subsection{Trigeminal Laser Stimulation}

\subsubsection{Pain Ratings}

For the right forehead condition, the subjective pain ratings (VAS scores) from the migraine group $(M=65.52, S D=18.90)$ and the pain ratings from the control group $(M=58.40, S D=$ 21.49) were not significantly different $(t=1.15, p=0.259)$.

\subsubsection{Laser-Evoked Potentials}

When plotting the grand average laser-evoked potential (LEP) separately for both groups at central electrodes, we see as expected from the literature the well-known N2 and P2 components in both groups (see Figure 1 left panel). Likewise, topographical plots at the N2 and $\mathrm{P} 2$ latencies show that these components are most pronounced around central electrodes (see Figure 1 right panel). 

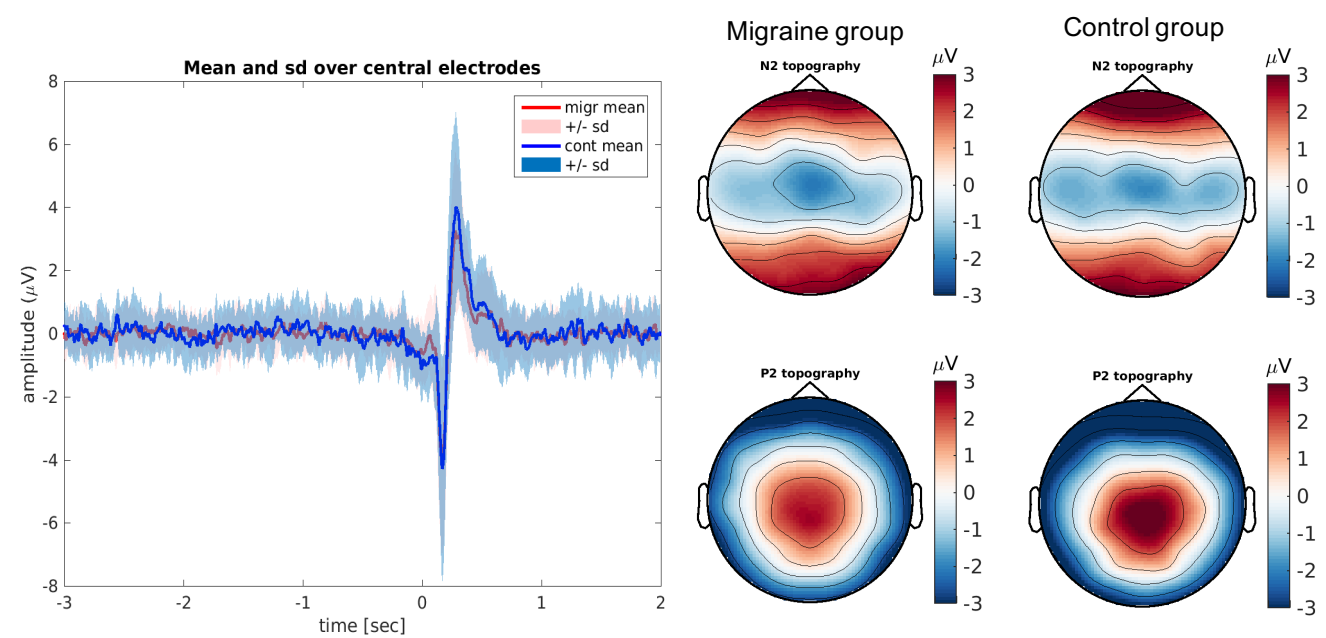

Figure 1. Group mean and standard deviation (sd) of laser-evoked potentials (LEPs) over central electrodes (left) and average topographical plots of N2 and P2 components (right) for the migraine and control group after right forehead laser stimulation.

\subsubsection{Time-frequency}

For the migraine group, at central electrodes, we found significant increases in the gammaband relative to baseline at $148 \mathrm{~ms} \mathrm{(Cz:} t=5.49, p=0.004 ; \mathrm{FC} 2: t=5.56, p=0.004$ and FCz: $t$ $=5.75, p=0.003)$ and $199 \mathrm{~ms}(\mathrm{Cz}: t=4.78, p=0.028)$. For the control group we saw a significant increase at one central electrode at $199 \mathrm{~ms}(\mathrm{C} 2: t=5.05, p=0.033)$ when correcting for multiple testing. We found no significant differences between groups when correcting for multiple testing (see Figure 2 for $t$-values). The average baseline GBOs at all 61 electrodes did not differ between groups (all $p$-values $>=0.59$ ). We saw significant positive Spearman correlations between the GBOs at central electrodes and the subjective pain ratings (see Figure 3 ) for the control group ( $r=0.44, t=2.09, p=0.027$ ) but not for the migraine group ( $r$ $=-0.08, t=-0.35, p=0.656)$. The positive Spearman correlation between central GBOs and migraine attack frequency was not significant $(r=0.22, t=1.01, p=0.304)$ but the negative correlation between central GBOs and years of migraine history was significant $(r=-0.45, t=$ $-2.33, p=0.034$ ). See Figure 9 for scatter plots displaying the associations between GBOs and the clinical features. 


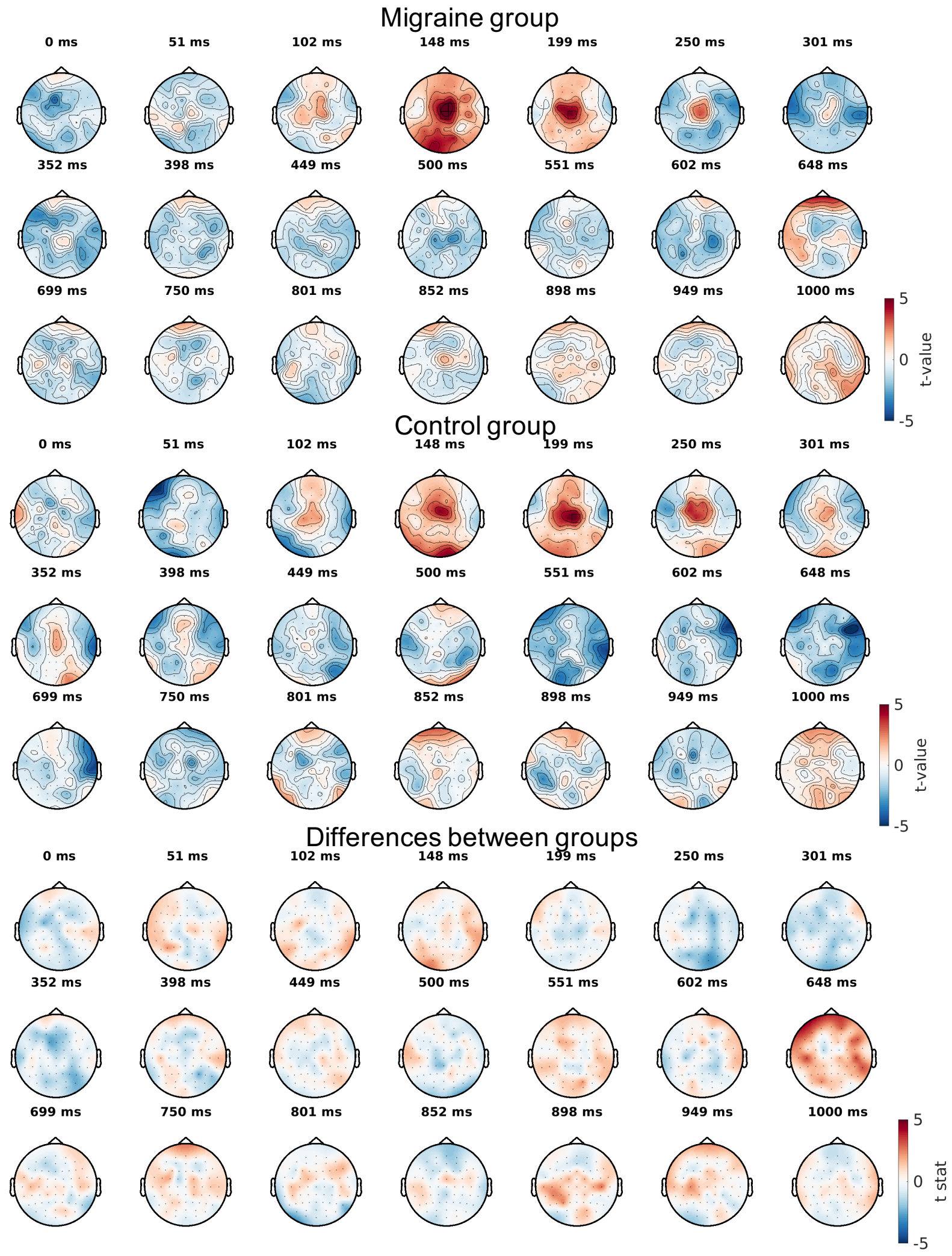

Figure 2. Gamma-band oscillations after right forehead laser stimulation. Upper panel: t-values from every electrode and every timeframe for the migraine group. Middle panel: $t$-values from every electrode and every timeframe for the control group. Bottom panel: $t$-values from the two-sample $t-$ tests comparing groups at every electrode and every timeframe. 

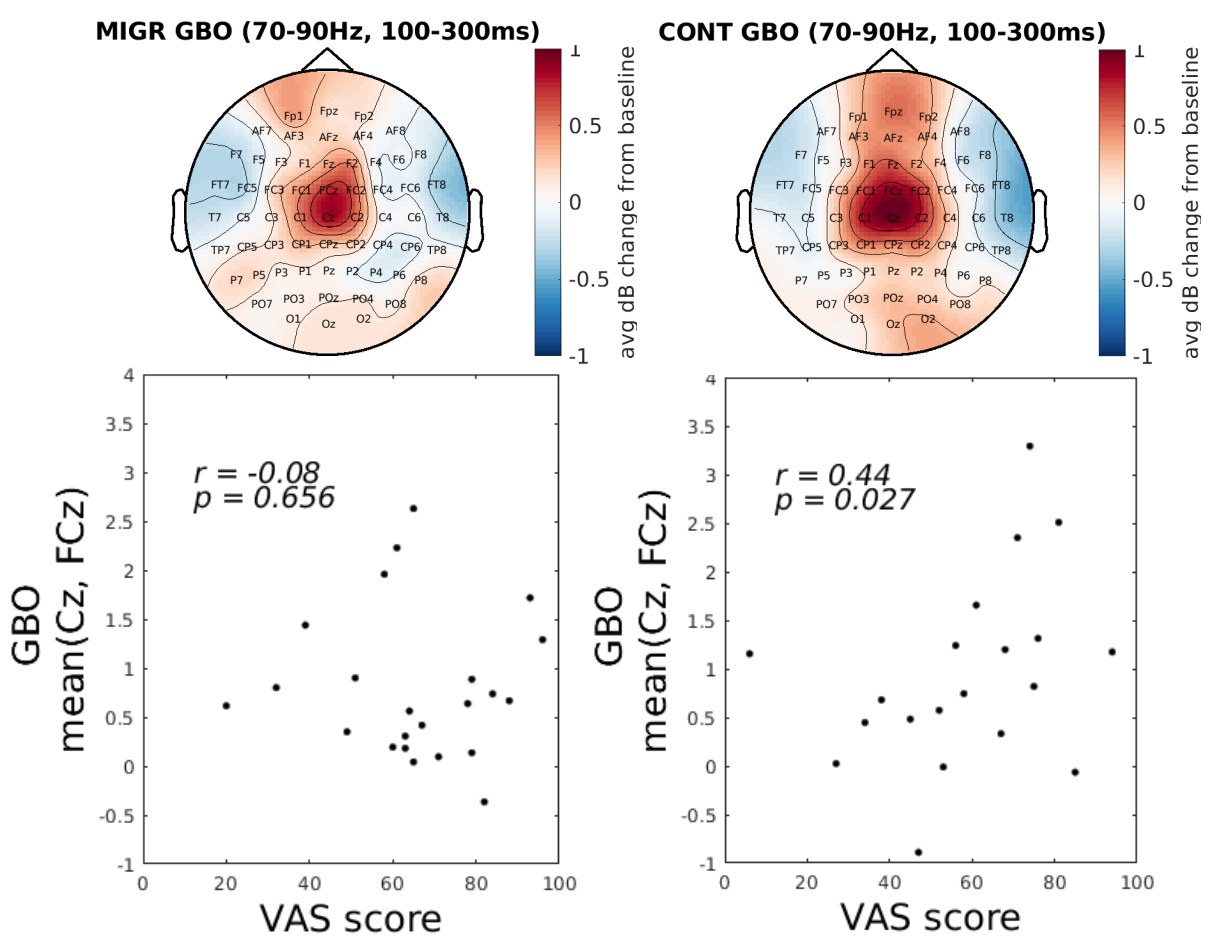

Figure 3. Topographical plots of average gamma-band increases $(70-90 \mathrm{~Hz}, 100-300 \mathrm{~ms})$ after right forehead laser stimulation in the migraine and control group and correlations of those gamma-band oscillations (GBOs) with pain ratings (VAS score) for both groups.

\subsubsection{Gamma sources}

For the migraine group, brain areas that included voxels with the $1 \%$ highest increases relative to baseline were the left and right midcingulate gyrus, left middle frontal gyrus, left superior frontal gyrus, left and right medial superior frontal gyrus, left and right supplementary motor area (see left panel Figure 4). Roughly the same regions showed increased activation in the control group, that is the left and right midcingulate gyrus, the right middle frontal gyrus, the left and right superior frontal gyrus, the left paracentral lobule, and the left and right supplementary motor area (see right panel Figure 4). MNI coordinates of the peak increase in the regions can be found in Table 1 . The power increases in the sources did not significantly differ between groups (see bottom panel Figure 4 for t-values). 


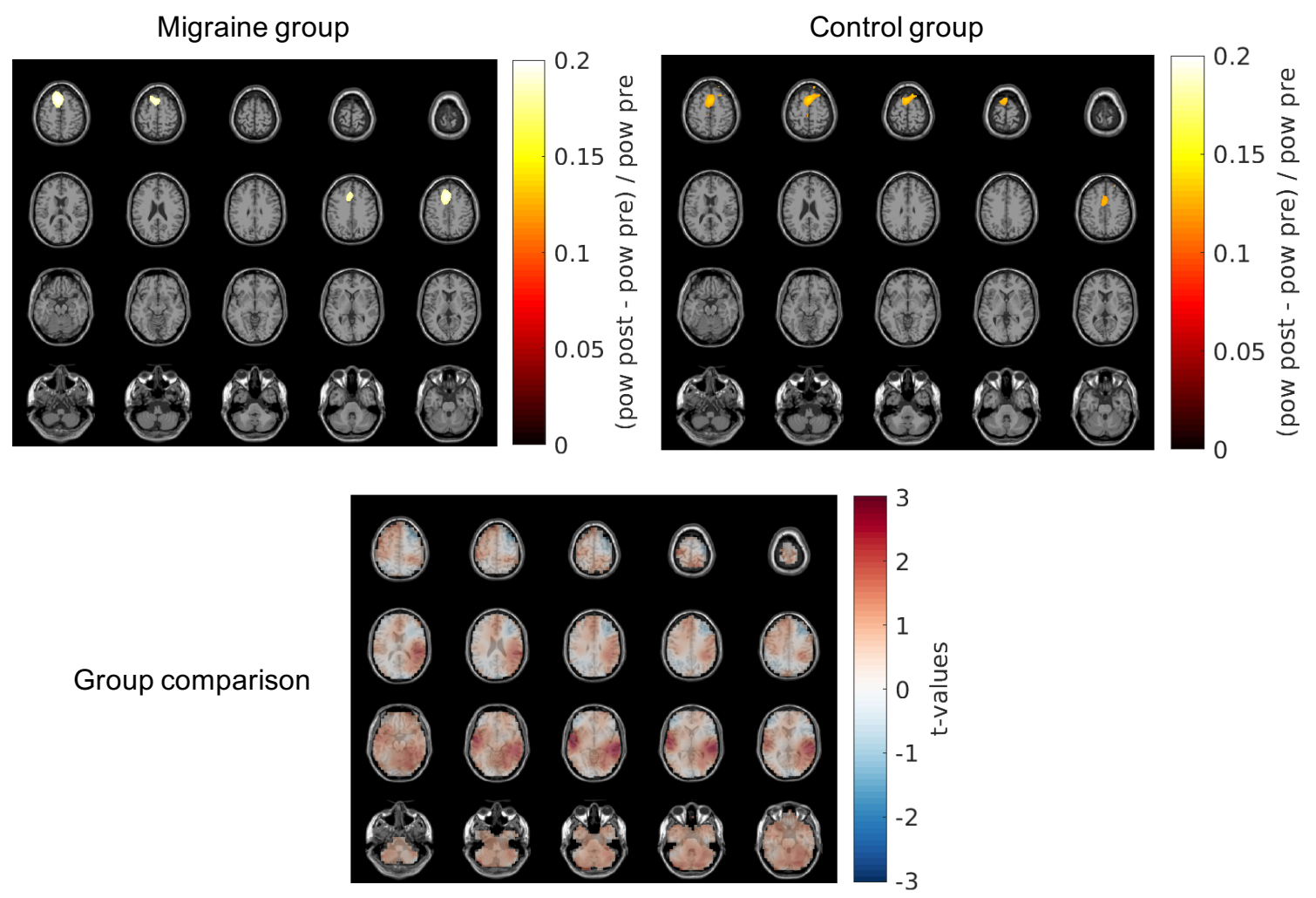

Figure 4. Beamforming results for the migraine group (left) and control group (right) after right forehead laser stimulation (upper panels). The voxels with the $1 \%$ highest power increases relative to baseline are highlighted. Bottom panel: $t$-values from the two-sample t-tests comparing groups.

\subsection{Hand Laser Stimulation}

\subsubsection{Pain Ratings}

The subjective pain ratings from the migraine group $(M=59.17, S D=18.65)$ and the control group $(M=53.87, S D=14.19)$ after right hand stimulation were not significantly different ( $t=$ $1.09, p=0.284)$.

\subsubsection{Laser-Evoked Potentials}

When plotting the grand average LEPs separately for both groups at central electrodes, we see as expected the well-known N2 and P2 components (see Figure 5 left panel). Likewise, plotting the N2 and P2 topography, we see that these components are most pronounced around central electrodes (see Figure 5 right panel). 

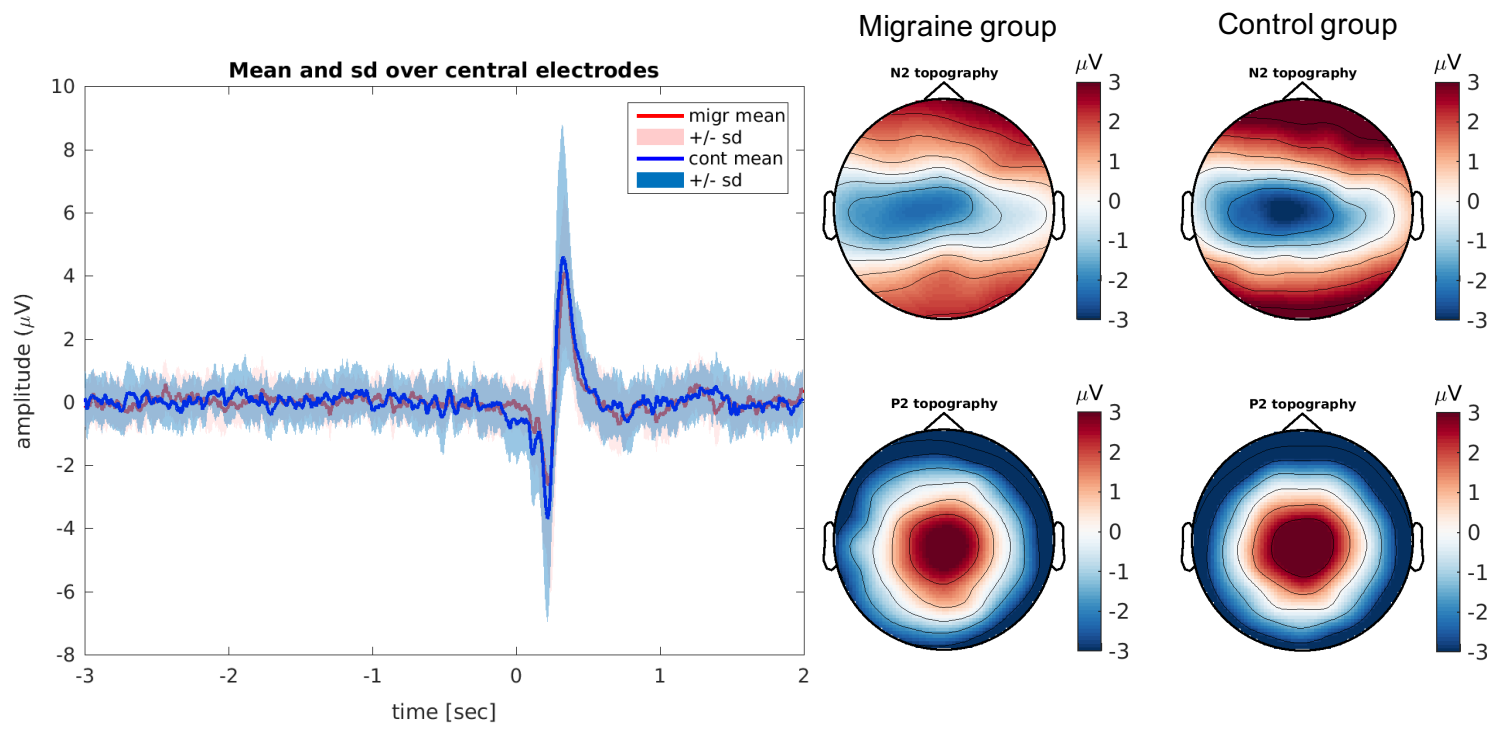

Figure 5. Group mean and standard deviation (sd) of laser-evoked potentials (LEPs) over central electrodes (left) and average topographical plots of N2 and P2 components (right) for the migraine and control group after right hand laser stimulation.

\subsubsection{Time-frequency.}

For the migraine group, we found significant increases in gamma-band activity, at $250 \mathrm{~ms}$ on electrode FC2 ( $t=4.92, p=0.022)$ and C2 $(t=5.03, p=0.016)$ and a significant increase at 250 $\mathrm{ms}$, at the $\mathrm{C} 2$ electrode for the control group ( $t=4.67, p=0.031)$. The increases in gamma activity were not significantly different between groups (see Figure 6 for topographical plots of $t$-values). Average baseline GBOs at all 61 electrodes, did not differ between groups (all $p$ values $>=0.58$ ). We observed a marginal significant positive correlation between the average gamma-band increases and the subjective pain intensities (see Figure 7) for the control group $(r=0.34, t=1.67, p=0.055)$ but not for the migraine group $(r=0.09, t=0.40, p=0.354)$. The positive Spearman correlation between GBOs and migraine attack frequency was not significant ( $r=0.26, t=1.24, p=0.222$ ) nor was the negative association between GBOs and years of migraine history $(r=-0.27 t=-1.30, p=0.195)$. See Figure 9 for scatter plots displaying the associations between GBOs and the clinical features. 


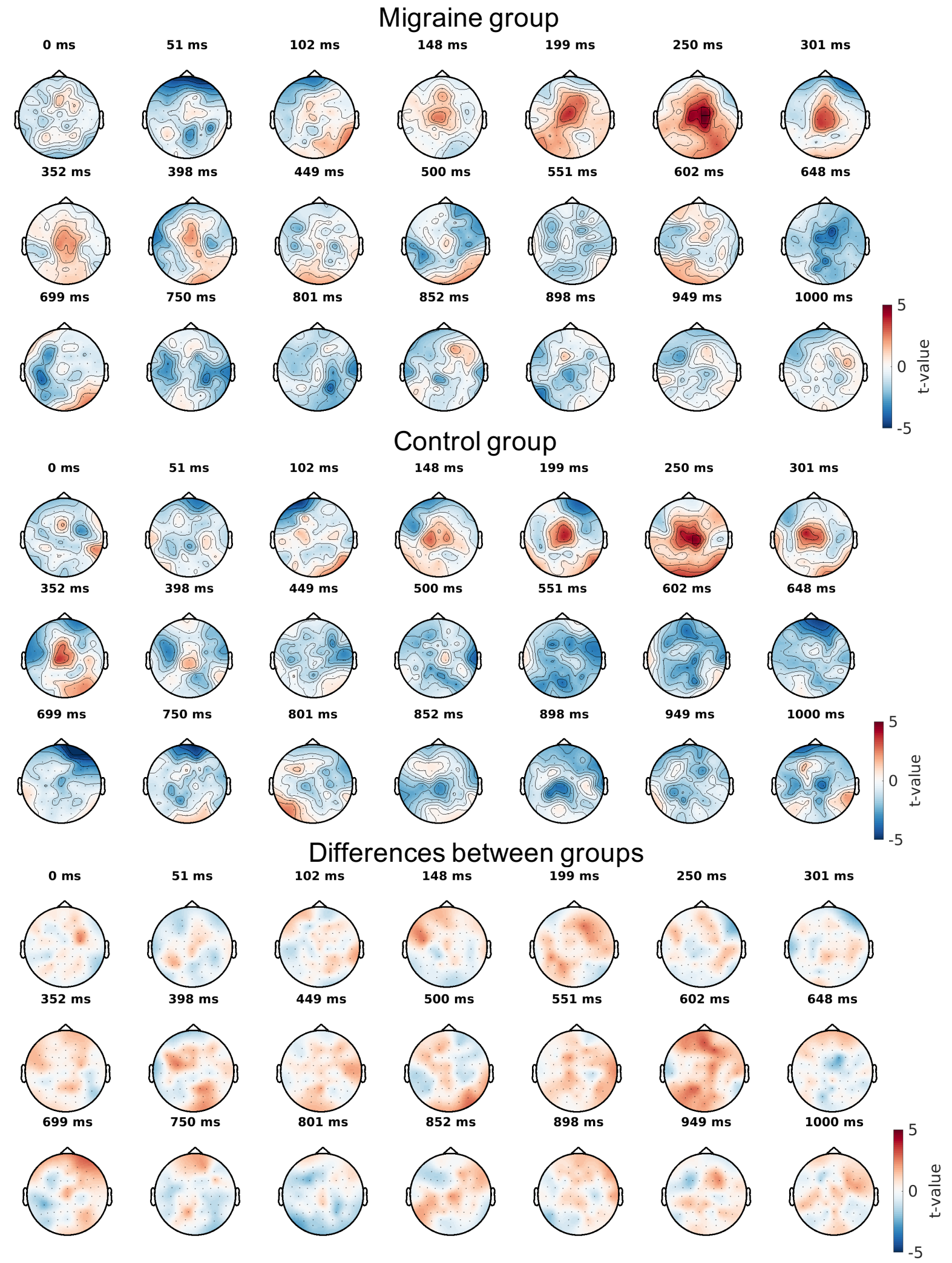

Figure 6. Gamma-band oscillations after right hand laser stimulation. Upper panel: t-values from every electrode and every timeframe for the migraine group. Middle panel: $t$-values from every electrode and every timeframe for the control group. Bottom panel: $t$-values from two-sample t-tests comparing groups at every electrode and every timeframe. 

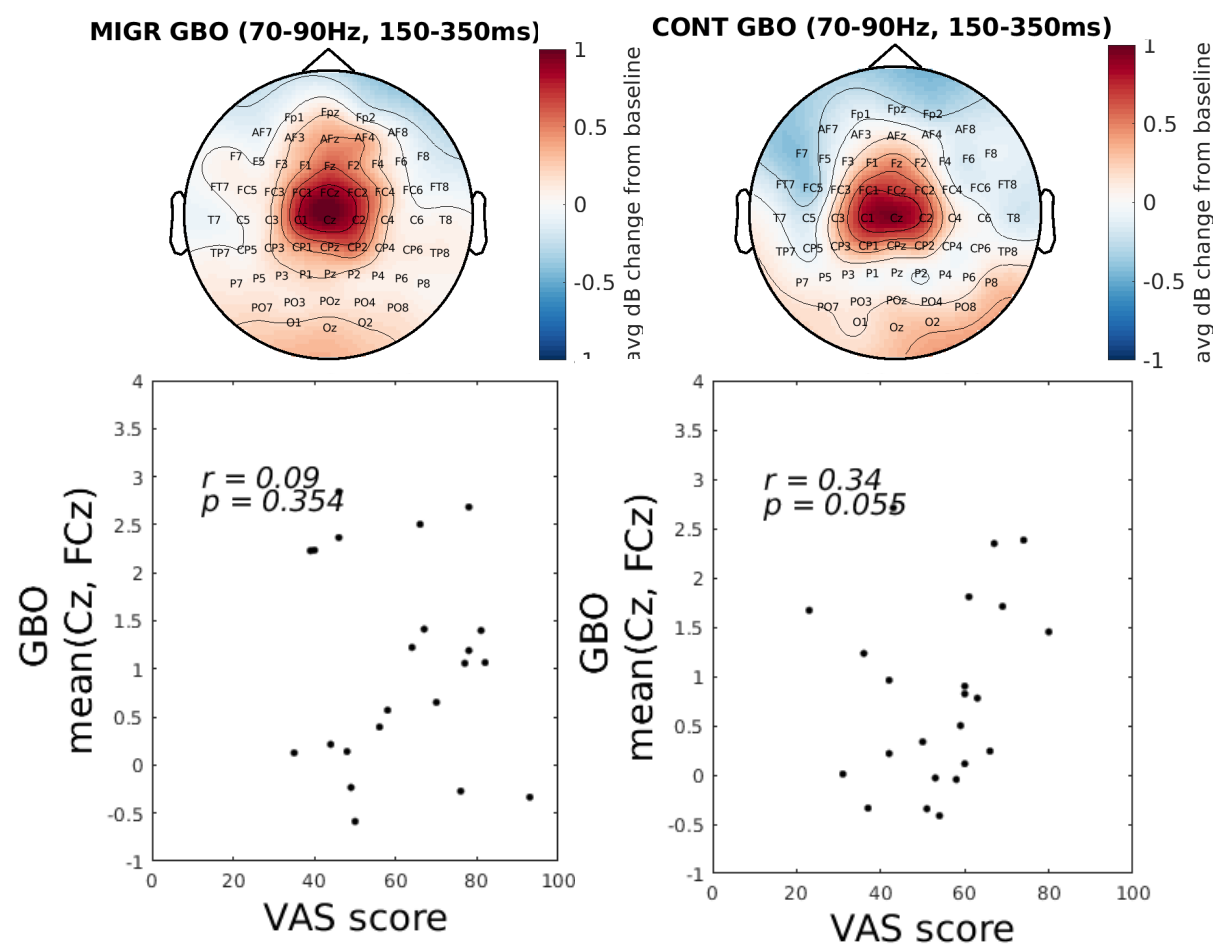

Figure 7. Topographical plots of average gamma-band increases $(70-90 \mathrm{~Hz}, 150-350 \mathrm{~ms})$ after right hand laser stimulation in the migraine and control group and correlations of those gamma-band oscillations (GBOs) with pain ratings (VAS score) for both groups.

\subsubsection{Gamma sources.}

For the migraine group, brain areas that included voxels with the $1 \%$ highest increases relative to baseline were the right caudate nucleus, the right midcingulate gyrus, right medial superior frontal gyrus, right superior frontal gyrus, right insula, right rolandic operculum and the right supplementary motor area (see left panel Figure 8 ). The $1 \%$ highest increases relative to baseline in the control group were the left calcarine, left and right midcingulate gyrus, left cuneus, left inferior parietal gyrus, right precuneus, left and right supramarginal gyrus and right middle temporal gyrus (see right panel Figure 8). MNI coordinates of the peak increase in the regions can be found in Table 1 . The power increases in the sources did not significantly differ between groups (see bottom panel Figure 8 for t-values). 


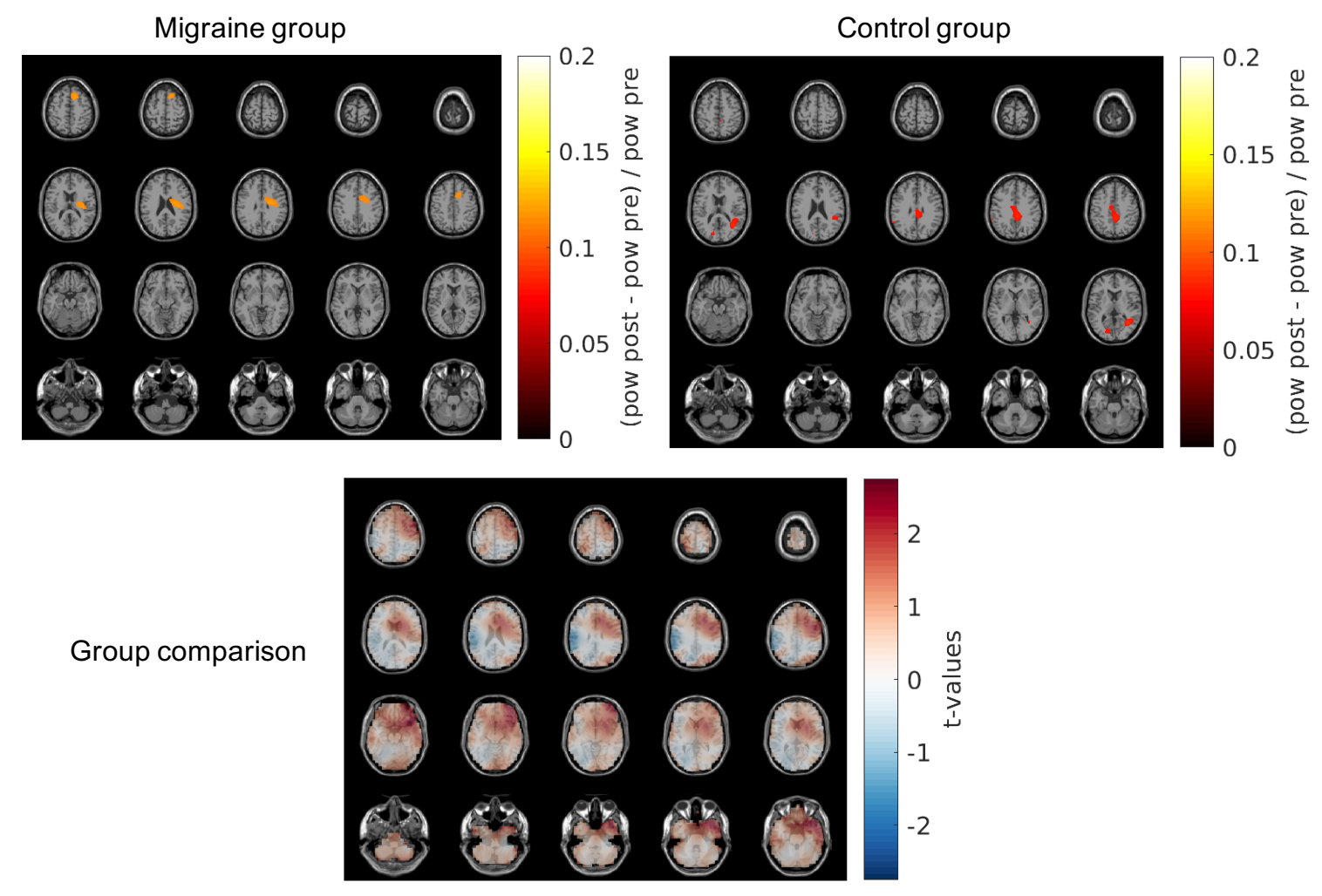

Figure 8. Beamforming results for the migraine group (left) and control group (right) after right hand laser stimulation (upper panels). The voxels with the $1 \%$ highest power increases relative to baseline are highlighted. Bottom panel: t-values from the two-sample t-tests comparing groups.
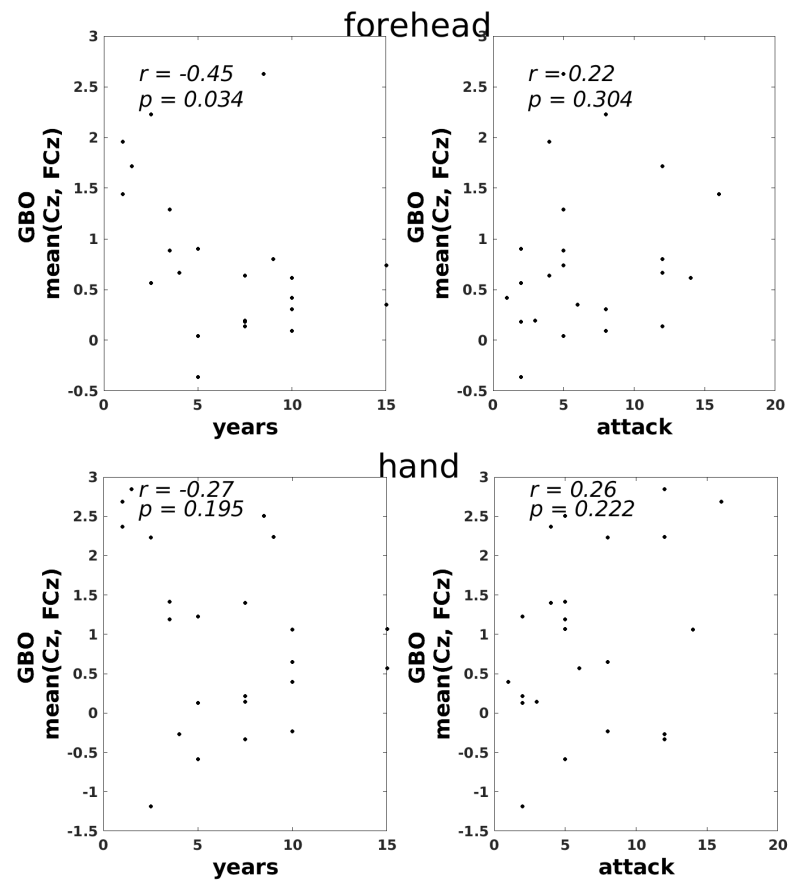

Figure 9. Scatter plots between gamma-band oscillations (GBOs) and years of migraine history and between GBOs and migraine attack frequency (in days) for the forehead and hand condition. 


\begin{tabular}{|c|c|c|c|c|}
\hline \multirow[t]{2}{*}{ Region (AAL parcel) } & \multicolumn{3}{|c|}{ MNI coordinates } & \multirow{2}{*}{$\begin{array}{c}\text { Percentage change } \\
\text { power }\end{array}$} \\
\hline & $x$ & $\mathrm{y}$ & z & \\
\hline \multicolumn{5}{|l|}{ Migraine group, forehead condition } \\
\hline Left midcingulate gyrus & -4 & 14 & 40 & $19,05 \%$ \\
\hline Right midcingulate gyrus & 4 & 22 & 40 & $18,72 \%$ \\
\hline Left supplementary motor area & -4 & 22 & 48 & $20,90 \%$ \\
\hline Right supplementary motor area & 4 & 22 & 48 & $19,89 \%$ \\
\hline Left medial superior frontal gyrus & -4 & 30 & 48 & $20,02 \%$ \\
\hline Right medial superior frontal gyrus & 4 & 30 & 48 & $18,56 \%$ \\
\hline Left middle frontal gyrus & -20 & 22 & 48 & $18,83 \%$ \\
\hline Left superior frontal gyrus & -12 & 22 & 56 & $20,30 \%$ \\
\hline \multicolumn{5}{|l|}{ Control group, forehead condition } \\
\hline Left midcingulate gyrus & -4 & -2 & 48 & $13,01 \%$ \\
\hline Right midcingulate gyrus & 4 & 6 & 40 & $12,32 \%$ \\
\hline Left supplementary motor area & -4 & 14 & 56 & $13,93 \%$ \\
\hline Right supplementary motor area & 4 & 14 & 56 & $14,47 \%$ \\
\hline Left superior frontal gyrus & -12 & 6 & 72 & $13,06 \%$ \\
\hline Right superior frontal gyrus & 20 & 22 & 64 & $13,09 \%$ \\
\hline Right middle frontal gyrus & 28 & 22 & 56 & $12,72 \%$ \\
\hline Left paracentral lobule & -4 & -26 & 56 & $12,36 \%$ \\
\hline \multicolumn{5}{|l|}{ Migraine group, hand condition } \\
\hline Right midcingulate gyrus & 12 & 6 & 40 & $11,69 \%$ \\
\hline Right supplementary motor area & 12 & 22 & 56 & $12,18 \%$ \\
\hline Right superior frontal gyrus & 20 & 22 & 56 & $12,07 \%$ \\
\hline Right medial superior frontal gyrus & 12 & 30 & 56 & $11,44 \%$ \\
\hline Right insula & 36 & -18 & 16 & $11,89 \%$ \\
\hline Righ caudate nucleus & 20 & -10 & 24 & $12,16 \%$ \\
\hline Right rolandic operculum & 44 & -26 & 16 & $11,64 \%$ \\
\hline \multicolumn{5}{|l|}{ Control group, hand condition } \\
\hline Left midcingulate gyrus & -4 & -10 & 40 & $8,35 \%$ \\
\hline Right midcingulate gyrus & 4 & -34 & 40 & $8,80 \%$ \\
\hline Left supramarginal gyrus & -60 & -50 & 32 & $8,87 \%$ \\
\hline Right supramarginal gyrus & 44 & -34 & 24 & $8,12 \%$ \\
\hline Right middle temporal gyrus & 44 & -50 & 16 & $8,26 \%$ \\
\hline Left cuneus & -12 & -82 & 16 & $8,23 \%$ \\
\hline Left calcarine & -12 & -82 & 8 & $8,06 \%$ \\
\hline Left inferior parietal gyrus & -60 & -42 & 40 & $8,30 \%$ \\
\hline Right precuneus & 12 & -42 & 40 & $8,34 \%$ \\
\hline
\end{tabular}

Note. Only one set of coordinates per region is shown (with maximum power increase). The automated anatomical labeling (AAL) atlas was used (Tzourio-Mazoyer et al., 2002)

\section{Discussion}

In this study it was investigated whether and when increases in GBOs can be found after painful laser stimulation in a group of episodic migraine patients without aura and a group of healthy controls and whether this differs between groups. In addition, it was examined whether these increases in GBOs after painful stimuli are related to pain perception, as found in previous research with healthy controls (Gross et al., 2007; Schulz et al., 2011b; Zhang et al., 2012), and to clinical features as migraine attack frequency and years of migraine history. 
To accomplish this, both groups received a series of laser stimuli on their right forehead and hand and after each series they were asked to indicate the perceived pain during that series.

In both groups, increases in GBOs were observed at central electrodes both after painful hand and forehead laser stimulation. Only in the control group, these increases seem to be related to subjective pain ratings. This finding in healthy controls is in accordance with previous studies (Gross et al., 2007; Schulz et al., 2011b; Zhang et al., 2012). Note that we cannot say anything about whether the GBOs here reflect pain perception rather than stimulus saliency as we did not manipulate this. We calculated the average time-frequency power over several trials (with most likely different saliencies) and correlated it with the pain rating which referred to the whole series. To our knowledge, this is the first study that investigated GBOs after painful stimuli in migraine patients. Unlike in healthy controls, we did not find significant correlations between central GBOs and subjective pain. As GBOs were not significantly different between groups, this might suggest that neurophysiological correlates of subjective pain in migraine patients are more complex. Positive associations between the gamma-band increases and the number of attacks a patient experiences per month were observed in both the hand and forehead condition, but these correlations were not significant. In contrast, years of migraine history was negatively related to GBOs, but this association was only significant in the forehead condition. In the current sample, there was a lot of variability in years of migraine history. When one would compare GBOs between a specific group of migraine patients (e.g., only patients with a long migraine history) and healthy controls different results could be found. Associations between years of migraine history (and migraine attack frequency) and atypical brain functionality in $\mathrm{FMRI}$ studies have led researchers to suggest that a cumulative effect of migraine exist on brain functionality (Schwedt et al. 2015). In light of present results, the correlation between GBOs and subjective pain perception, may vary with history of migraine and probably with other chronic conditions. Future studies with larger sample sizes should try to investigate this.

When looking at the sources of the gamma-band power increases, we saw that the midcingulate cortex was consistently observed over the two conditions and two groups. In all conditions, except the hand condition from the control group, we also found the supplementary motor areas and other frontal regions. Other studies found increases in GBOs after painful laser stimuli in the sensorimotor cortex (Gross et al., 2007; Tiemann et al., 2010) and the insula (Liberati et al., 2018a, 2018b). Gross et al. (2007) used MEG and found GBOs in the primary somatosensory cortex. However, the authors selected somatosensory cortices based on maximum pain-evoked activity and then exclusively looked at GBOs in these regions, thus not excluding the possibility of pain induced GBOs in other regions. Pain-evoked activity is also present in the cingulate cortex (Garcia-Larrea et al., 2003). Note that there are differences in sensitivity for specific sources between EEG and MEG (Ahlfors et al., 2010). In line with our results, a recent study that used a similar beamforming method, contrasted low and high laser pain intensities and found generators of pain-induced gamma oscillations to be 
in the sensorimotor and midcingulate cortex (Hauck et al., 2015). Further, it's not unexpected that cingulate, supplementary motor area and frontal regions were observed here as these have been found to be related to nociception (Apkarian et al., 2005; Peyron et al., 2000). So has activation in the midcingulate region been found to be positively related to pain intensity (Büchel et al., 2002). It is also suggested that the cingulate cortex plays an orienting role in pain processing (Peyron et al., 2000). This affective-attentional role of the cingulate region could be in accordance with a recent hypothesis mentioned by Ploner et al. (2017) where it is proposed that GBOs may be important in feedforward information transfer. Hauck et al. (2015), who also found GBOs in the midcingulate cortex, suggests that GBOs may be important in the information flow of signals related to pain, which make the GBOs important to integrate the input of noxious stimuli into an overall pain experience. Overall, the source findings are not contradictory to present theories of pain-related GBOs and pain processing in general.

Finally, there has been some discussion about GBOs being neural or muscular as these two high-frequency sources are often hard to disentangle (Muthukumaraswamy, 2013). We chose to use a preprocessing approach that automatically corrects (or rejects) artifactual data (ASR) and automatically rejects artifactual independent components (MARA) as we want our results to be reproducible and independent on the expertise and subjective eye of the data analyst. Although the methods have been shown to perform well in muscle artifact removal, to the best of our knowledge there is currently no method to eliminate all muscle artifacts with complete certainty. Still, several reasons support that the found GBOs here may have a neural origin. First, the spatial maps show maximum gamma-band increases at central electrodes while a spatial distribution with maximum increases at electrodes near the edges (parietooccipital, frontal and temporal) would be more indicative for muscle artifacts (Muthukumaraswamy, 2013). Second, we see that the spatial and temporal properties of the GBOs are similar to the spatial and temporal properties of the well-known LEPs which could support that both are related to nociceptive processing. Finally, the source localization method (beamforming) reveals GBOs in the midcingulate cortex, a region that has been consistently found to be involved in nociceptive processing. A study that wanted to separate neural from muscular activity in the context of visual-induced gamma-band activity found that such a beamforming method allowed separating neural activity from extracranial muscle artifacts (Hipp et al., 2013).

To conclude, GBOs induced by laser stimulations were present at central electrodes but these were not significantly different between interictal migraine and control. We found that central GBOs were associated with pain perception in healthy individuals, in accordance with other studies. Interestingly, no evidence for such an association in the migraine group was found. Finally, we suggest the midcingulate cortex to be a source of nociceptive-related GBOs. 


\section{Acknowledgements}

This work was supported by the University of Bari Aldo Moro research funds and the Fund for Scientific Research-Flanders (FWO-V, PhD Fellowship grant number FW017/ASP/042 awarded to IB; http://www.fwo.be).

\section{Declaration of Competing Interest}

None of the authors have potential conflicts of interest to be disclosed.

\section{Author Contributions}

IB contributed to data and statistical analysis, manuscript preparation and editing. KR, EV, MD, and EG contributed to neurophysiological assessment and data acquisition. DM contributed to decisions on analysis methods and manuscript editing. MdT contributed to conception and study design, clinical assessment, and manuscript preparation and editing. All authors contributed to revisions of the manuscript and approved the final version. 


\section{References}

Ahlfors, S. P., Han, J., Belliveau, J. W., \& Hämäläinen, M. S. (2010). Sensitivity of MEG and EEG to source orientation. Brain Topogr., 23, 227-232. https://doi.org/10.1007/s10548010-0154-x

Apkarian, A. V., Bushnell, M. C., Treede, R.-D., \& Zubieta, J.-K. (2005). Human brain mechanisms of pain perception and regulation in health and disease. Eur. J. Pain, 9(4), 463-463. https://doi.org/10.1016/j.ejpain.2004.11.001

Büchel, C., Bornhovd, K., Quante, M., Glauche, V., Bromm, B., \& Weiller, C. (2002). Dissociable neural responses related to pain intensity, stimulus intensity, and stimulus awareness within the anterior cingulate cortex: A parametric single-trial laser functional magnetic resonance imaging study. J. Neurosci., 22(3), 970-976. https://doi.org/10.1523/jneurosci.22-03-00970.2002

Chang, C.-Y., Hsu, S.-H., Pion-Tonachini, L., \& Jung, T.-P. (2019). Evaluation of artifact subspace reconstruction for automatic artifact components removal in multi-channel EEG recordings. IEEE Trans. Biomed. Eng. https://doi.org/10.1109/tbme.2019.2930186

Cohen, M. X. (2014). Analyzing Neural Time Series Data. MIT press.

Colombo, B., Rocca, M. A., Messina, R., Guerrieri, S., \& Filippi, M. (2015). Resting-state fMRI functional connectivity: A new perspective to evaluate pain modulation in migraine? Neurol. Sci., 36, 41-45. https://doi.org/10.1007/s10072-015-2145-x

de Tommaso, M., Ambrosini, A., Brighina, F., Coppola, G., Perrotta, A., Pierelli F. et al. (2014). Altered processing of sensory stimuli in patients with migraine. Nat. Rev. Neurol., 10(3), 144-155. https://doi.org/10.1038/nrneurol.2014.14

Delorme, A., \& Makeig, S. (2004). EEGLAB: An open source toolbox for analysis of single-trail EEG dynamics including independent component anlaysis. J. Neurosci. Methods, 134, 921. https://doi.org/10.1016/j.jneumeth.2003.10.009

Garcia-Larrea, L., Frot, M., \& Valeriani, M. (2003). Brain generators of laser-evoked potentials: From dipoles to functional significance. Neurophysiol. Clin. Neurophysiol., 33, 279-292. https://doi.org/10.1016/j.neucli.2003.10.008

Goadsby, P. J., Holland, P. R., Martins-Oliveira, M., Hoffmann, J., Schankin, C., \& Akerman, S. (2017). Pathophysiology of migraine: A disorder of sensory processing. Physiol. Rev., 97(2), 553-622. https://doi.org/10.1152/physrev.00034.2015

Gross, J., Kujala, J., Hämäläinen, M., Timmermann, L., Schnitzler, A., \& Salmelin, R. (2001). Dynamic imaging of coherent sources: Studying neural interactions in the human brain. PNAS, 98, 694-699. https://doi.org/10.1073/pnas.98.2.694

Gross, J., Schnitzler, A., Timmermann, L., \& Ploner, M. (2007). Gamma oscillations in human primary somatosensory cortex reflect pain perception. PLoS Biol., 5(5), 1168-1173. https://doi.org/10.1371/journal.pbio.0050133

Hauck, M., Domnick, C., Lorenz, J., Gerloff, C., \& Engel, A. K. (2015). Top-down and bottomup modulation of pain-induced oscillations. Front. Hum. Neurosci., 9. https://doi.org/10.3389/fnhum.2015.00375

Hipp, J. F., Siegel, M., \& Deouell, L. Y. (2013). Dissociating neuronal gamma-band activity from cranial and ocular muscle activity in EEG. Front. Hum. Neurosci., 7. https://doi.org/10.3389/fnhum.2013.00338

lannetti, G. D., Hughes, N. P., Lee, M. C., \& Mouraux, A. (2008). Determinants of laser-evoked EEG responses: Pain perception or stimulus saliency? J. Neurophysiol., 100(2), 815-828. https://doi.org/10.1152/jn.00097.2008

IHS. (2013). The International Classification of Headache Disorders, 3rd edition (beta 
version). Cephalalgia, 33(9), 629-808. https://doi.org/10.1177/0333102413485658

IHS. (2018). The International Classification of Headache Disorders, 3rd edition. Cephalalgia, 38(1), 1-211. https://doi.org/10.1177/0333102417738202

Leske, S., \& Dalal, S. S. (2019). Reducing power line noise in EEG and MEG data via spectrum interpolation. Neuroimage, 189, 763-776.

https://doi.org/10.1016/j.neuroimage.2019.01.026

Liberati, G., Algoet, M., Klöcker, A., Ferrao Santos, S., Ribeiro-Vaz, J. G., Raftopoulos, C. et al. (2018b). Habituation of phase-locked local field potentials and gamma-band oscillations recorded from the human insula. Sci. Rep., 8. https://doi.org/10.1038/s41598-01826604-0

Liberati, G., Klöcker, A., Algoet, M., Mulders, D., Safronova, M. M., Ferrao Santos, S. et al. (2018a). Gamma-band oscillations preferential for nociception can be recorded in the human insula. Cereb. Cortex, 28(10), 3650-3664.

https://doi.org/10.1093/cercor/bhx237

Mullen, T. R., Kothe, C. A. E., Chi, M., Ojeda, A., Kerth, T., Makeig, S. et al. (2015). Real-time neuroimaging and cognitive monitoring using wearable dry EEG. IEEE Trans Biomed Eng, 62(11), 2553-2567. https://doi.org/10.1109/tbme.2015.2481482

Muthukumaraswamy, S. D. (2013). High-frequency brain activity and muscle artifacts in MEG/EEG: A review and recommendations. Front. Hum. Neurosci., 7. https://doi.org/10.3389/fnhum.2013.00138

Oostenveld, R., Fries, P., Maris, E., \& Schoffelen, J.-M. (2011). FieldTrip: Open source software for advanced analysis of MEG, EEG, and invasive electrophysiological data. Comput. Intell. Neurosci., 2011. https://doi.org/10.1155/2011/156869

Peyron, R., Laurent, B., \& García-Larrea, L. (2000). Functional imaging of brain responses to pain. A review and meta-analysis. Clin. Neurophysiol., 30(5), 263-288. https://doi.org/10.1016/s0987-7053(00)00227-6

Ploner, M., Sorg, C., \& Gross, J. (2017). Brain Rhythms of Pain. Trends Cogn. Sci., 21(2), 100110. https://doi.org/10.1016/j.tics.2016.12.001

Ronga, I., Valentini, E., Mouraux, A., \& lannetti, G. D. (2013). Novelty is not enough: Laserevoked potentials are determined by stimulus saliency, not absolute novelty. J. Neurophysiol., 109(3), 692-701. https://doi.org/10.1152/jn.00464.2012

Schulz, E., Tiemann, L., Schuster, T., Gross, J., \& Ploner, M. (2011a). Neurophysiological coding of traits and states in the perception of pain. Cereb. Cortex, 21(10), 2408-2414. https://doi.org/10.1093/cercor/bhr027

Schulz, E., Tiemann, L., Witkovsky, V., Schmidt, P., \& Ploner, M. (2012). Gamma oscillations are involved in the sensorimotor transformation of pain. J. Neurophysiol., 108(4), 10251031. https://doi.org/10.1152/jn.00186.2012

Schulz, E., Zherdin, A., Tiemann, L., Plant, C., \& Ploner, M. (2011b). Decoding an individual's sensitivity to pain from the multivariate analysis of EEG data. Cereb. Cortex, 22, 11181123. https://doi.org/10.1093/cercor/bhr186

Schwedt, T. J., Chiang, C. C., Chong, C. D., \& Dodick, D. W. (2015). Functional MRI of migraine. Lancet Neurol., 14(1), 81-91. https://doi.org/10.1016/s1474-4422(14)701930

Stankewitz, A., Schulz, E., \& May, A. (2013). Neuronal correlates of impaired habituation in response to repeated trigemino-nociceptive but not to olfactory input in migraineurs: An fMRI study. Cephalalgia, 33(4), 256-265.

https://doi.org/10.1177/0333102412470215 
Tiemann, L., Schulz, E., Gross, J., \& Ploner, M. (2010). Gamma oscillations as a neuronal correlate of the attentional effects of pain. Pain, 150, 302-308.

https://doi.org/10.1016/j.pain.2010.05.014

Treede, R.-D., Lorenz, J., \& Baumgärtner, U. (2003). Clinical usefulness of laser-evoked potentials. Clin. Neurophysiol., 33, 303-314.

https://doi.org/10.1016/j.neucli.2003.10.009

Tzourio-Mazoyer, N., Landeau, B., Papathanassiou, D., Crivello, F., Etard, O., Delcroix, N. et al. (2002). Automated Anatomical Labeling of activations in SPM using a macroscopic anatomical parcellation of the MNI MRI single-subject brain. Neuroimage, 15, 273-289. https://doi.org/10.1006/nimg.2001.0978

Valeriani, M., De Tommaso, M., Restuccia, D., Le Pera, D., Guido, M., lannetti, G. D. et al. (2003). Reduced habituation to experimental pain in migraine patients: A CO 2 laser evoked potential study. Pain, 105, 57-64. https://doi.org/10.1016/s03043959(03)00137-4

Winkler, I., Haufe, S., \& Tangermann, M. (2011). Automatic classification of artifactual ICAcomponents for artifact removal in EEG signals. Behav. Brain Funct., 7(30). https://doi.org/10.1186/1744-9081-7-30

Zhang, Z. G., Hu, L., Hung, Y. S., Mouraux, A., \& lannetti, G. D. (2012). Gamma-band oscillations in the primary somatosensory cortex: A direct and obligatory correlate of subjective pain intensity. J. Neurosci., 32(22), 7429-7438.

https://doi.org/10.1523/jneurosci.5877-11.2012 